\title{
HABCO: Herramienta informática para la automatización de la calificación de exámenes para apoyo al docente
}

\author{
Luis Javier Mona Peña \\ Instituto Tecnológico de Saltillo, Departamento de Ingeniería Eléctrica-Electrónica, \\ México \\ lmona@itsaltillo.edu.mx
}

\begin{abstract}
Resumen. El desarrollo de software es una herramienta poderosa para auxiliar al hombre en múltiples áreas de su quehacer cotidiano. En áreas de evaluación del nivel cognitivo, de aptitudes, de desempeño entre otros, es especialmente útil ya que existen herramientas de medición diseñadas para que por medio de exámenes de opción múltiple cuantificar dicho nivel cognitivo y demás habilidades y competencias.El presente artículo se enfoca al uso del software LabVIEW (Laboratory Virtual Instrumentation Engineering Workbench) en el desarrollo de una herramienta capaz de auxiliar a los profesionales que utilizan dichas pruebas en la evaluación y calificación de las mismas de una manera sencilla, rápida, eficiente, confiable, con rastreabilidad de la información y flexible para cualquier formato de examen de alveolos impreso. El software es flexible en el aspecto de que no importa el formato utilizado (número de alveolos, opciones disponibles de respuesta, distribución geométrica) de las hojas de respuesta de las pruebas, ya que solamente se requiere entrenar una vez al sistema con las respuestas correctas. Las hojas de respuestas de las pruebas son digitalizadas por medio de un escáner para después utilizar algoritmos de procesamiento digital de imágenes (Visión por Computadora) para realizar el análisis con el propósito de verificar respuestas correctas y acto seguido obtener la ponderación de cada pregunta las cuales están en una base de datos de SQL Server. Una vez obtenido resultados estos se exportan a un formato en Microsoft Excel para el posterior manejo de la información obtenida.
\end{abstract}

Palabras clave: Alveolo, excel, LabVIEW, SQL, visión.

\section{HABCO: Computer Tool for Automatic Exam Evaluation to Help a Teacher}

\begin{abstract}
Software development is a powerful tool to help in many areas of the men daily work. In areas such as evaluation of cognitive level, skills, performance, among others, it is particularly useful because there are measurement tools designed in order to use multiple-choice tests for measuring cognitive level and other skills and competencies. This article focuses on the use
\end{abstract}




\begin{abstract}
of software LabVIEW (Laboratory Virtual Instrumentation Engineering Workbench) to develop a tool to assist professionals who use these tests in evaluating and rating them in a simple, fast, efficient, and reliable way, with traceability information and flexible for holding any kind of printed multiple choice test format. The software is flexible in the way that doesn't matter the format used (number of alveoli, response options, geometric distribution) of the test's answer sheets, since it is only required to train once the software system with the correct answers. The test's answer sheets are digitized by a scanner and then use algorithms of digital image processing (Machine Vision) for analysis in order to verify correct answers and immediately obtain the weighting of each question which are in a database in SQL Server. After obtaining these results the data are exported to a Microsoft Excel format for further handling of the information obtained.
\end{abstract}

Keywords: Alveoli, excel, LabVIEW, SQL, vision.

\title{
1. Introducción
}

El uso de la tecnología ha potenciado la aplicación de técnicas y herramientas que permiten obtener información sobre diversos aspectos de la naturaleza y en específico del hombre mismo y su entorno. En el caso del ramo educativo, existen herramientas para evaluar y calificar diferentes áreas de conocimiento, aptitudes y habilidades tales como la prueba ENLACE (Evaluación Nacional del Logro Académico en Centros Escolares) y el examen del CENEVAL (Centro Nacional de Evaluación). Estas pruebas están diseñadas para que el presentante responda a los reactivos por medio de alveolos (opción múltiple), la evaluación y calificación de dichos exámenes se realiza por medio de herramientas de software que permite tener en pocos minutos el resultado de miles de exámenes. En el caso del docente, se tiene libertad sobre el diseño y aplicación de sus propios exámenes, sin embargo la labor de evaluación y calificación se puede tornar desgastante debido a la cantidad de preguntas y el número de alumnos a los que se les aplica la prueba, llegando a dedicar horas enteras a la evaluación y calificación al realizar estas actividades de forma manual por no tener una herramienta que les auxilie en dichas tareas.

El reto principal era desarrollar una herramienta por software altamente confiable y fácil de usar para la evaluación y calificación automática de exámenes de respuestas tipo alveolo que fuera universal, es decir, que no importara la cantidad de preguntas, el número de opciones de respuesta, el formato de distribución, el tamaño de la hoja, etc. Además que fuera capaz de guardar la información en una base de datos en SQL Server los datos del presentante, la ponderación individual de cada reactivo, la calificación final y poder cambiar rápidamente la ponderación de los reactivos modificando los valores en una tabla en la base de datos. El sistema es capaz de detectar si más de una opción por reactivo es seleccionada en el caso de tratar de engañar al sistema y tener tolerancia a los patrones e intensidad del rellenado de la opción del reactivo. Por último, realizar el reporte de resultados y exportarlo a formato de Microsoft Excel para que el usuario final realizara las operaciones estadísticas que considere necesarias. 


\section{Metodología}

Para la implementación de la aplicación se definió la siguiente metodología la cual sigue en orden secuencial las etapas de acondicionamiento, obtención, análisis y presentación de la información:

1. Digitalización de las hojas de respuesta de la pruebas. Esta etapa es la que alimenta al sistema, requiere que el usuario por medio de un escáner digitalice las pruebas y las guarde en el formato de su preferencia, siendo recomendable los formatos .jpg y .png por la relación de calidad-tamaño de archivo que presentan. Cada archivo se guarda en disco con el nombre del presentante que corresponda y se almacena en una carpeta que se llama "Exámenes" que es una carpeta que el sistema define por default para la búsqueda de las imágenes para su proceso.

2. Calibración del sistema. Dentro de calibración de sistema se definen las regiones de búsqueda que serán utilizadas para determinar por medio de algoritmos de procesamiento de imágenes del toolkit de Machine Vision del software LabVIEW 2015 de National Instruments si las respuestas son correctas o no. La información de las coordenadas de las regiones de búsqueda tanto de intensidad de pixeles como de búsqueda de patrones se guardan en disco duro utilizando un formato de archivo binario para aumentar la velocidad de acceso a la información y la eficiencia en el guardado de la misma.

3. Conexión con la base de datos en SQL Server Express 2014. Se define una base de datos relacional con dos tablas. Una de ellas de nombre "Alumnos" tiene diversos campos como Nombre, un campo por reactivo donde se pondrá la ponderación lograda y otro campo con la suma de todas ponderaciones o calificación general por alumno. Una segunda tabla "Ponderaciones" tiene un campo por cada reactivo donde el usuario especifíca el valor de ponderación, de esta forma se logra flexibilidad de la aplicación, ya que el usuario puede cambiar a su criterio las ponderaciones de los reactivos en el momento que lo deseé sin que tenga que programar nada.

4. Análisis de las imágenes. Determinar la cantidad de respuestas correctas según el Master realizado en la etapa de calibración de sistema se utilizan algoritmos de conteo de pixeles e intensidad de nivel de grises necesarios para corroborar si la opción que el presentante seleccionó es la correcta o no. En esta misma etapa se utilizan algoritmos de búsqueda de patrones con herramientas del toolkit de Machine Vision, esta búsqueda de patrones tiene como objetivo determinar que el presentante no haya seleccionado más de una opción por reactivo lo cual podría ser una forma engañar el sistema, por lo tanto si existe más de una respuesta seleccionada el sistema determinará que la respuesta es incorrecta a pesar del resultado del análisis de pixeles.

5. Actualización de la Información en la base de datos. La información obtenida por medio de los algoritmos del análisis de imágenes es actualizada en la base de datos dentro de la tabla "Alumnos" donde queda respaldada para su consulta posterior.

6. Generación del reporte final de calificaciones. Etapa que genera el reporte final de calificaciones a nivel local y remoto. La información proveniente del 
análisis de la información es utilizada para generar una tabla ASCII que se visualiza dentro de la misma interfaz de la aplicación donde cada renglón es el registro del alumno y cada columna la ponderación del reactivo que corresponda. Además por medio de conectividad ActiveX se genera un reporte en Microsoft Excel para mayor portabilidad de la información generada además de ser una herramienta que la mayoría de los docentes o profesionales utilizan para el análisis estadístico.

\section{Implementación de la aplicación.}

El software que se utilizó para el desarrollo del sistema de software es National Instruments LabVIEW 2015 ya que integra herramientas de Procesamiento Digital de Imágenes, Conectividad a bases de datos y ActiveX que son las herramientas seleccionadas en la metodología para la implementación del desarrollo de software. Además el programa es bajo el estilo de programación basada en eventos, esto para eficientar el uso de los recursos de hardware y software de la computadora que aloje la aplicación, ya que solo requerirá la atención de ésta cuando el usuario interactúe con el programa sin necesidad de realizar rutinas cíclicas (pulldown) que requieren gran parte de la atención del microprocesador.

El primer paso después de que las imágenes ya están digitalizadas es abrirlas para su análisis. El nombre de cada archivo debe corresponder al nombre del alumno, esto con el propósito de que el software tome el nombre del alumno directamente del archivo y lo guarde en la base datos para mayor comodidad del usuario. El algoritmo toma el nombre del archivo para actualizar la tabla "Alumnos" en el campo "Nombre" de la base de datos y crear los registros con los nombres de los alumnos que corresponde a cada examen. Esto se realiza utilizando comandos SQL. Cada imagen requiere una referencia para su uso la cual es creada por el subvi IMAQ Create del toolkit Vision Development System. La figura 1 ilustra el algoritmo completo de creación de referencias a las imágenes, obtención del nombre del archivo y la actualización en el campo "Nombre" de la tabla "Alumnos".

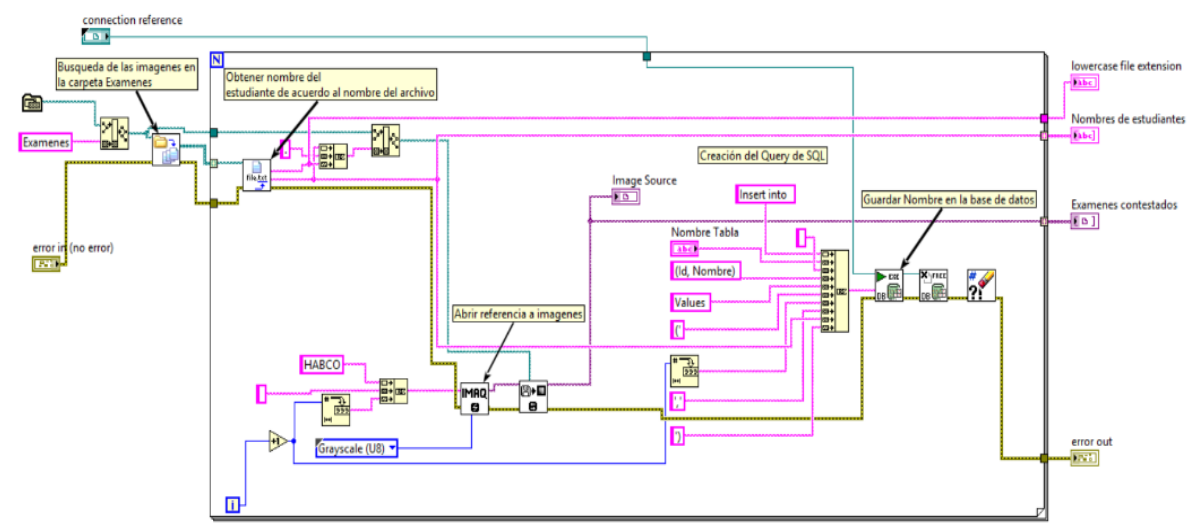

Fig. 1. Obtención de referencias de imágenes y obtención para guardado automático de los nombres de los alumnos. 
HABCO: Herramienta informática para la automatización de la calificación de exámenes ...

Este subvi (sub instrumento) da como salida arreglos con la información que requerirán los elementos posteriores para el análisis de las imágenes. El icono de dicho subvi se muestra en la figura 2.

\section{numero imagenes de los examens contestados.vi}

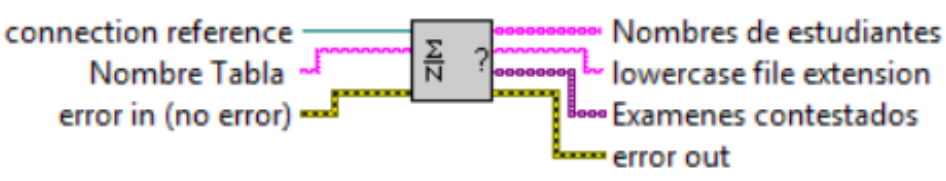

Fig. 2. Icono del Subvi de obtención de referencias de imágenes.

Ya con la referencia de las imágenes, se crean las regiones de búsqueda (ROIS Descriptors) de las respuestas correctas, ver figura 3. Son dos regiones de búsqueda, la primera de ellas se dibuja sobre la opción de la respuesta correcta, esta región es utilizada por el sistema para medir la intensidad de los pixeles buscando la tonalidad negra indicativa que se ha rellenado la opción. La segunda región de búsqueda se utiliza para buscar el patrón de respuesta rellena y por lo tanto obscura, esto se implementa como medida de seguridad necesaria para evitar que se seleccione más de una opción y que el criterio único del conteo de pixeles quede rebasado.

\section{COMUNICACIÓN}
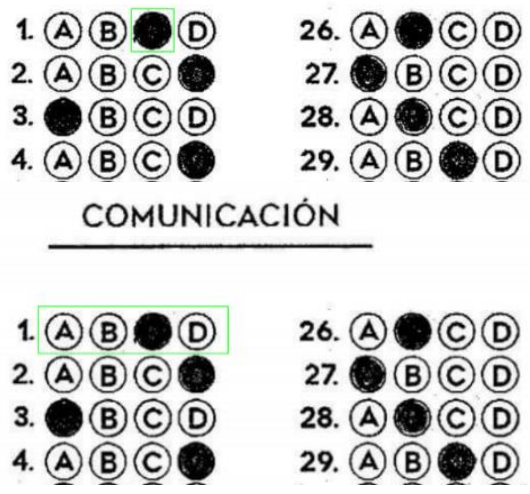

Fig. 3. Determinación de regiones de búsqueda de respuesta correcta y respuesta única.

De esta manera, si más de una opción es seleccionada, no importa si entre ellas va la respuesta correcta, el sistema lo considerará como pregunta mal contestada. La figura 4 muestra el código de implementación de la creación de las regiones de interés que serán utilizadas para la búsqueda de intensidad de pixeles y de patrones.

La información de las coordenadas de las regiones de interés es almacenada en dos arreglos de clusters que indican la descripción del rectángulo de búsqueda. Dicho cluster incluye la coordenada superior, la inferior y la rotación del rectángulo que define la región de interés o de búsqueda. Esta información se guarda en disco duro en formato 
binario y sirve como calibración del examen Master para efectuar la evaluación de los exámenes.

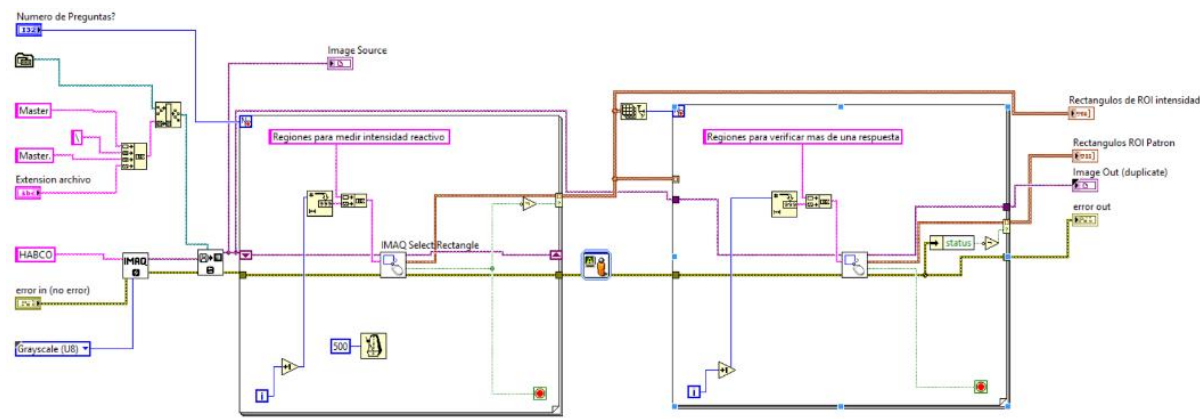

Fig. 4. Código de implementación de regiones de interés del sistema.

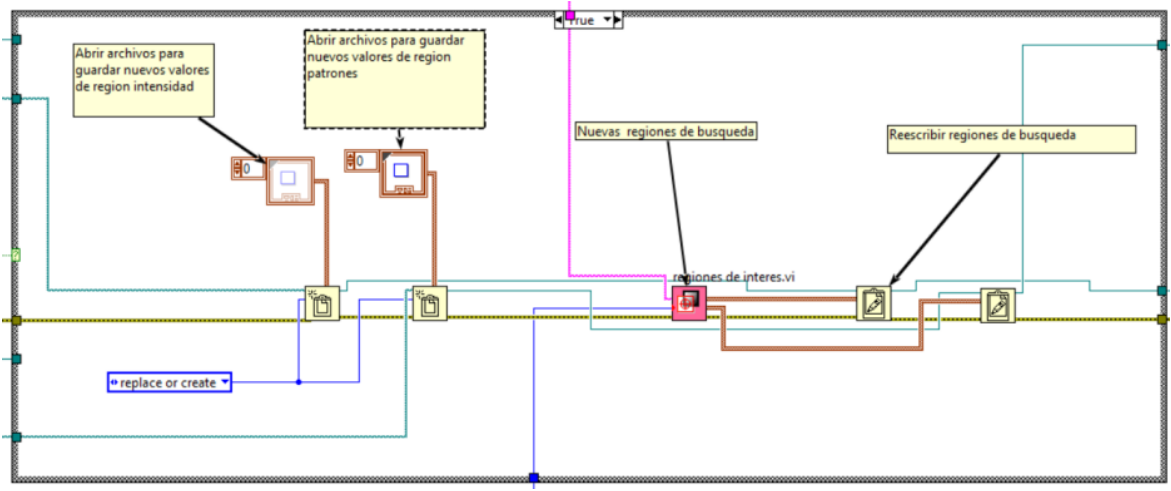

Fig. 5. Código para guardado de información de las regiones de interés en disco duro.

Ya definidas las regiones de interés, el sistema tiene toda la información para realizar la evaluación de los exámenes al buscar en primer lugar la intensidad de los pixeles (niveles de escala de grises) y en segundo término la búsqueda de patrones. La búsqueda de intensidad de pixeles tiene como propósito corroborar que el alumno escogió la respuesta correcta al repintar la opción seleccionada quedando de un color obscuro comparado con el resto de las opciones, tal como se muestra en la figura 3.

Dentro de las herramientas del módulo de visión de LabVIEW el subvi IMAQ Light Meter (1) realiza el conteo de la escala de grises en un rango de 0 a 255, siendo el blanco extremo un valor de 255 indicando la ausencia de pigmentaciones y el negro con un valor de 0 para el negro intenso. La figura 6 indica el código que implementa el subvi IMAQ Ligth Meter.

La salida de este subvi son valores estadísticos de la intensidad de escala de grises, siendo el valor promedio Mean Value el parámetro que utiliza el sistema para determinar si el usuario eligió la opción correcta. Utilizando las regiones de interés previamente obtenidas, se evalúa cada imagen (examen) obteniendo el valor promedio en la escala de grises pregunta por pregunta. El resultado es un arreglo bidimensional que contiene la información de cada examen junto con el conjunto de valores de 
HABCO: Herramienta informática para la automatización de la calificación de exámenes ...

medición de pixeles de cada pregunta. El código que se muestra en la figura 7 indica el código implementado para realizar la comprobación.

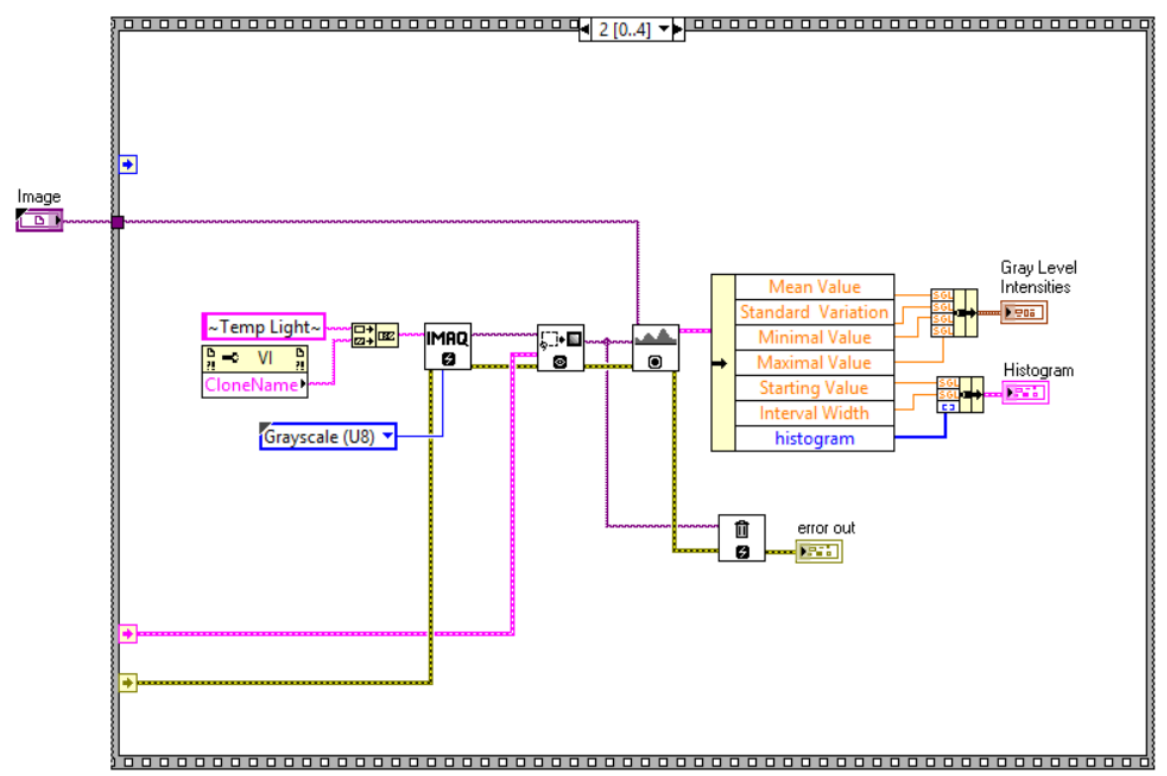

Fig. 6. Código del subvi IMAQ Ligth Meter.

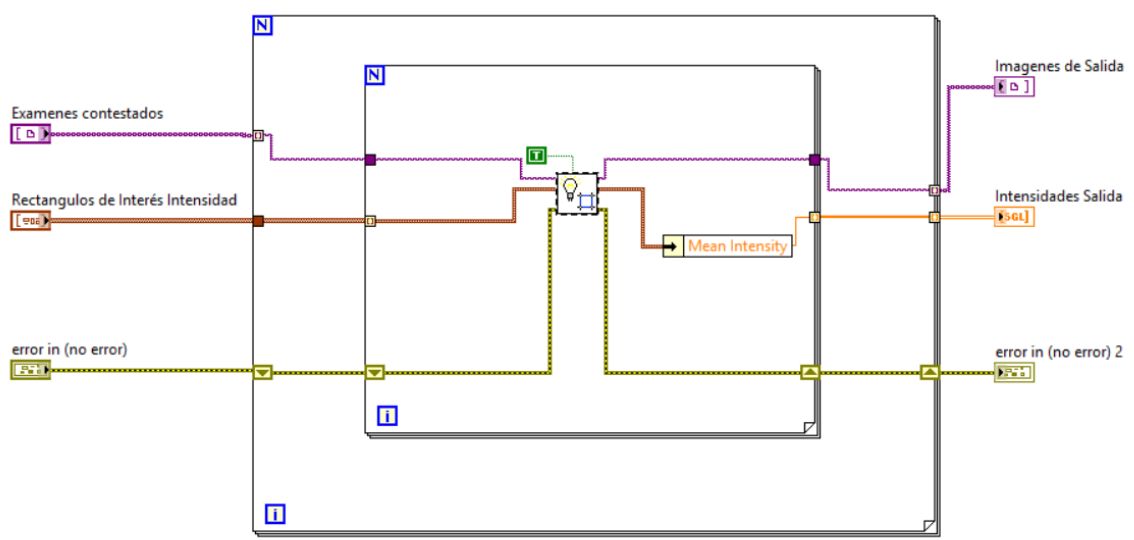

Fig. 7. Obtención de los valores de intensidad de pixeles de cada examen.

Para la comprobación de respuesta única por pregunta del examen se realiza una búsqueda del patrón de respuesta contestada, que sería la opción repintada. Esta acción se realiza con el algoritmo IMAQ Learn Pattern del módulo de visión de LabVIEW (2) donde realiza una búsqueda de una imagen patrón sobre una región de búsqueda en otra imagen, dando como resultado la cantidad de patrones encontrados. Este resultado se 
almacena en un arreglo bidimensional que tiene la información de a que examen corresponde cada una de las preguntas junto con el número de patrones encontrados. La cantidad máxima de patrones encontrados por pregunta debe de ser uno, ya que de lo contrario estamos hablando de preguntas con múltiples respuestas seleccionadas. La figura 8 muestra el código implementado para determinar la cantidad de opciones seleccionadas por pregunta de cada examen.

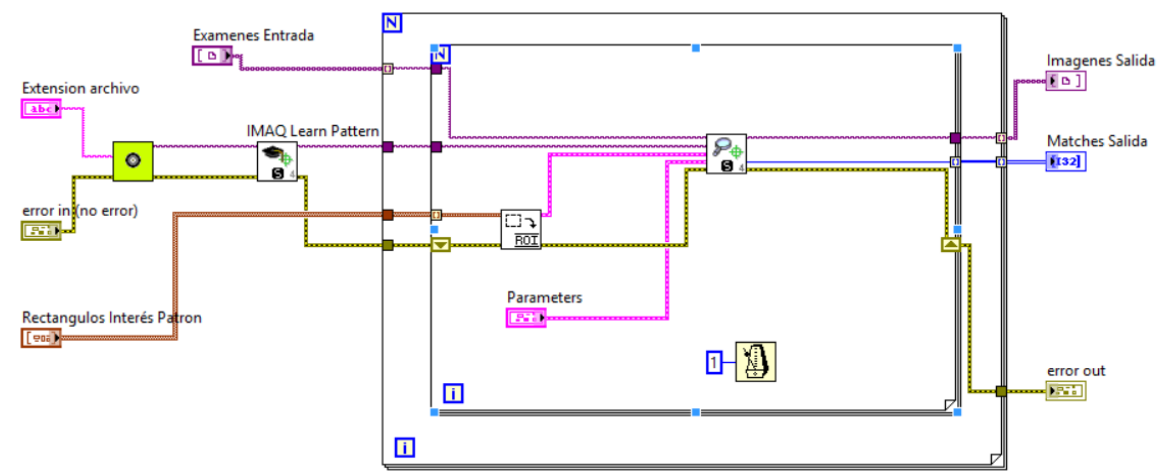

Fig. 8. Código de determinación de respuesta única.

Una vez que el software determina si las respuestas son correctas pasa a la etapa de cuantificación, es decir obtener de la base de datos el puntaje de cada una de las preguntas que colabora para la calificación final. Las ponderaciones de cada respuesta se encuentran en una tabla de nombre "Ponderaciones" de la base de datos de nombre HABCO implementada en SQL Server Express 2014 que utiliza el software, el diseño de la tabla se ilustra en la figura 9 .

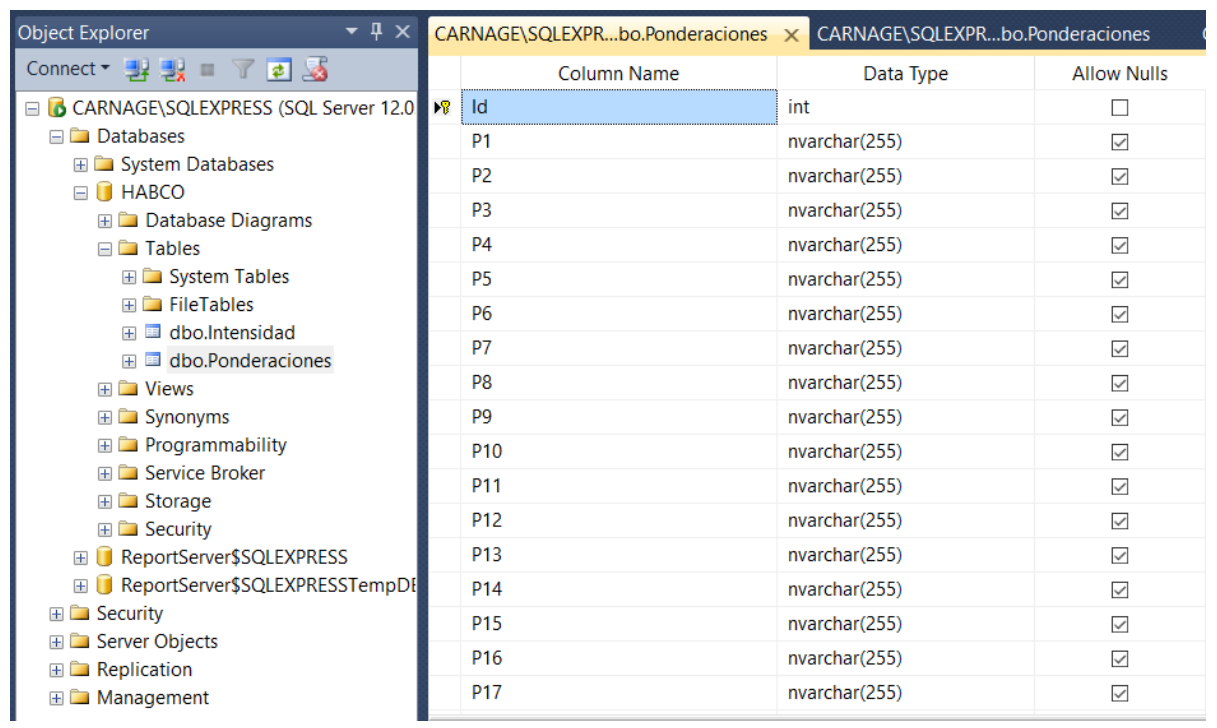

Fig. 9. Vista de diseño en SQL Server Express 2014 de la tabla ponderaciones. 
En esta tabla el usuario puede cambiar las ponderaciones de cada pregunta para cuestiones de actualización de las pruebas.

El código que implementa la búsqueda de la tabla "Ponderaciones" utiliza enunciados en SQL para buscar el valor de ponderación de cada reactivo.

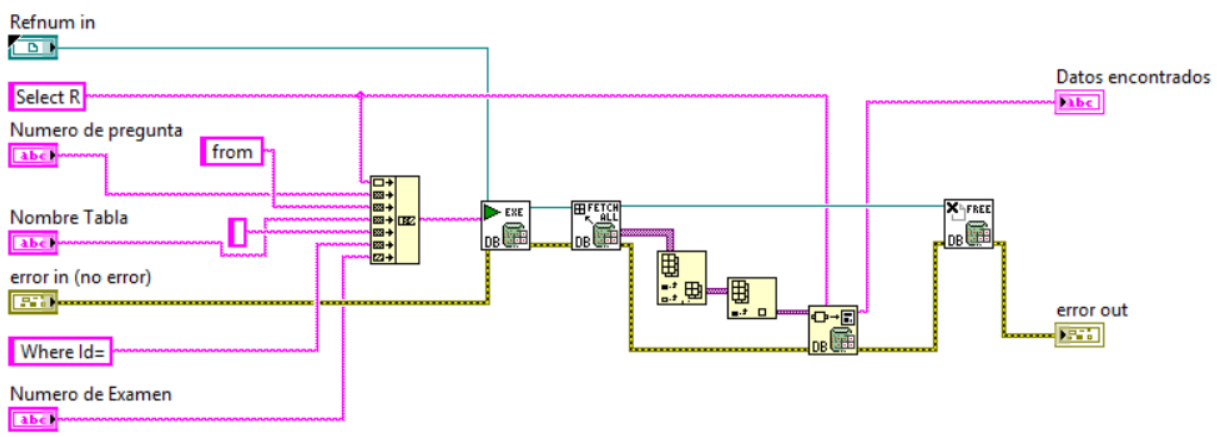

Fig. 10. Código para búsqueda de la ponderación de cada reactivo.

Ya con los valores de ponderación el sistema procede a actualizar cada campo que representa una pregunta del examen (Figura 11), dando el valor de cero cuando la respuesta es incorrecta y el valor correspondiente al valor de ponderación que se leyó de la tabla "Ponderaciones" cuando la respuesta es correcta. La herramienta del toolkit de conectividad a bases de datos Execute Query (3) permite ejecutar comandos SQL para la interacción con la base de datos.

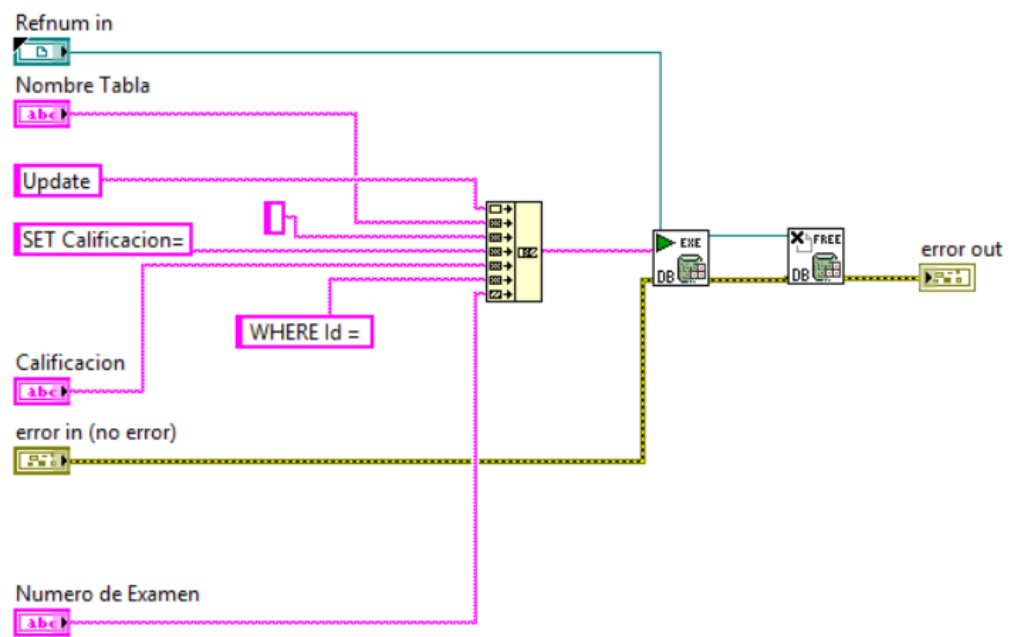

Fig. 11. Actualización de los campos de cada pregunta en la Tabla Alumnos en SQL Server Express 2014.

De la misma manera por medio de comandos en SQL (4) se realiza la tarea de actualizar campos de calificaciones, los cuales son los de la tabla "Alumnos" donde quedarán los registros con las calificaciones de cada examen teniendo como 
identificador del registro el nombre del alumno. Un ejemplo de cómo queda la tabla Alumnos de la base de datos del sistema se muestra en la figura 12 en su interfaz de SQL Server.

\begin{tabular}{|c|c|c|c|c|c|c|c|c|c|c|c|c|c|c|c|c|c|}
\hline \multirow{2}{*}{ 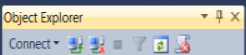 } & \multicolumn{17}{|c|}{ CARNAGESSQLEXPRE_.-doolintensidad X } \\
\hline & Id & Nombre & R1 & R2 & R3 & R4 & R5 & R6 6 & RT & R8 & $\mathrm{Rg}$ & R10 & R11 & $\mathbb{R} 12$ & R13 & R14 & R15 \\
\hline 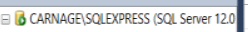 & 1 & Ana Lucia Martinez Reta & 0 & $a$ & 0 & 0 & 0 & 0 & $a$ & 0 & 0 & 0 & a & 0 & 12 & $a$ & 0 \\
\hline \multirow{2}{*}{$\begin{array}{l}\text { 日曰 Dattabases } \\
\text { 田 System Databases }\end{array}$} & 2 & Ana Paula larrazäbaal López & 0 & 0 & 6 & 12 & 12 & 9 & 0 & 0 & 0 & 9 & $a$ & 0 & 0 & 12 & 0 \\
\hline & 3 & Carlos Alberto Gomez Hemañndez & 6 & 9 & 0 & 0 & 12 & 9 & 0 & 6 & 6 & 0 & 18 & 0 & 0 & 12 & 12 \\
\hline \multirow{2}{*}{$\begin{array}{l}\text { 日 U HABCO } \\
\text { 田 Database Diagrams. }\end{array}$} & & Duke María Treviñó Leal & 0 & 9 & 0 & 0 & 12 & 9 & 6 & 0 & 6 & 0 & $a$ & 0 & 0 & 0 & 0. \\
\hline & 5 & José Dibilidox Hassaf & 0 & 9 & 6 & 12 & 12 & 0 & 0 & 0 & 6 & 0 & a & 0 & 0 & a & 0 \\
\hline $\begin{array}{l}\text { 日 Tables } \\
\text { 由】 System Tables }\end{array}$ & 6 & Josê Emiliano Moncada Quintanilla & 0 & a & 0 & 0 & 0 & 9 & 0 & 0 & 0 & 9 & a & 0 & 0 & 12 & 12 \\
\hline \multirow{2}{*}{ 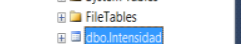 } & 7 & Liza Regina Cornejo Valdez & 0 & a & 0 & 0 & 0 & 0 & 0 & 0 & 0 & 0 & a & 0 & 0 & 12 & 12 \\
\hline & 8 & Marian Alejandra Luna Hernández & 0 & a & 0 & 0 & 0 & 9 & 6 & 0 & 0 & 0 & $a$ & 0 & 0 & $a$ & 0 \\
\hline 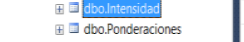 & 10 & Miriam Cabello Vallarta & 0 & 9 & 0 & 0 & 0 & 0 & 0 & 6 & 0 & 9 & 0 & 0 & 12 & 0 & 0 \\
\hline 町 Views & 11 & Roberto Sosa Davila & 0 & a & 0 & 0 & 0 & 9 & 6 & 0 & 6 & 9 & 0 & 0 & 0 & 0 & 0 \\
\hline 田 Programmability & 12 & Ximena Sofía Morales Padron & 6 & 9 & 6 & 12 & 12 & 9 & 6 & 6 & 6 & 9 & 0 & 0 & 12 & $a$ & 0 \\
\hline \multirow{2}{*}{$\begin{array}{l}\text { 田 Senvice Broker } \\
\text { ⿴囗十 Storage }\end{array}$} & 13 & Luis Adriàn Dogart de la Paz & 6 & 0 & 0 & 0 & 12 & g & 0 & 0 & 6 & 9 & 18 & 18 & 0 & 12 & 0 \\
\hline & 14 & Mariana Sánchez Muñoz & 0 & 0 & 0 & 0 & 0 & 0 & 0 & 0 & 0 & 9 & $a$ & 0 & 12 & 12 & 12 \\
\hline 田 Security & 15 & Scarlett lara Valennuela & 0 & 9 & 6 & 12 & 12 & 9 & 0 & 0 & 0 & 9 & 0 & 0 & 0 & 12 & 12 \\
\hline \multirow{3}{*}{ 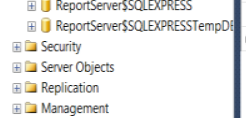 } & 16 & Ximena Villarreal Valdezz & 0 & 9 & 6 & 12 & 12 & 0 & 0 & 0 & 6 & 0 & 0 & 0 & 0 & 12 & 0 \\
\hline & \#. NULL & NULL & NULL & MULL & NULL & NUL & NuLL & NULL & NULL & nUL & nuil & NULL & NULL & nULL & NULL & NULL & NULL \\
\hline & & & & & & & & & & & & & & & & & \\
\hline
\end{tabular}

Fig. 12. Tabla Alumnos actualizada en SQL Server Express desde LabVIEW.

La calificación final es el resultado de la suma de todas las ponderaciones de los reactivos, dicha información se realiza con el siguiente código que involucra el promedio de los elementos del arreglo de calificaciones y la actualización en el campo "Calificación Final" de la tabla Alumnos. La figura 13 indica el código implementado.

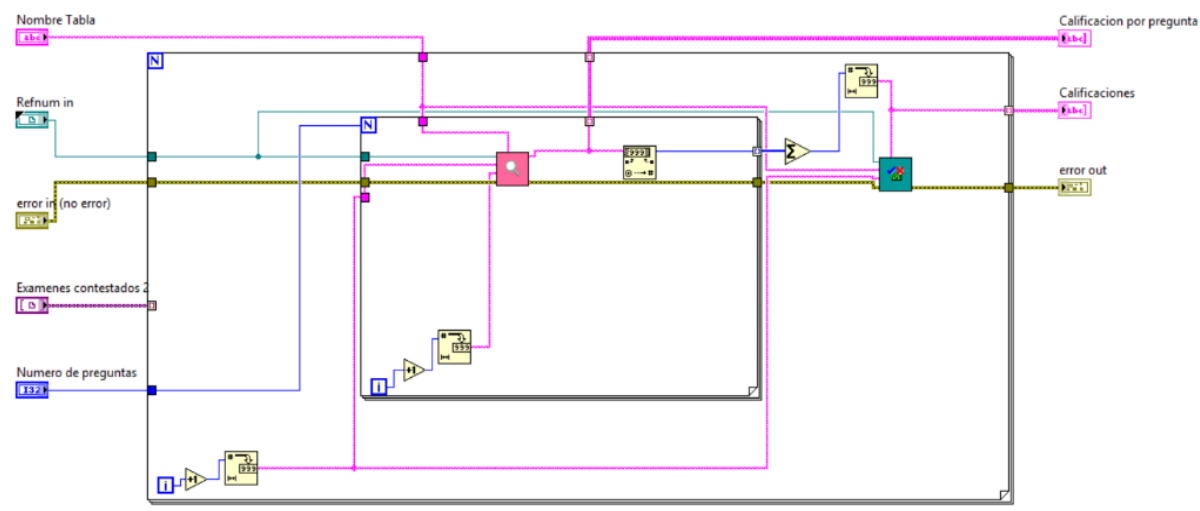

Fig. 13. Código para la obtención de la calificación final.

Finalmente la creación del reporte en Microsoft Excel se implementó utilizando el toolkit de Report Generation (5) de LabVIEW que a través de referencias ActiveX logra la comunicación y conectividad requerida. Dentro de LabVIEW se personalizó el tipo de reporte que incluye toda la información que se encuentra en la tabla Alumnos de la base de datos, es decir, Nombre del alumno, calificación por pregunta y calificación final. La Figura 14 ejemplifica parte del código implementado para la creación del reporte final de calificaciones del examen. El cual queda listo para su posterior análisis. 
HABCO: Herramienta informática para la automatización de la calificación de exámenes ...

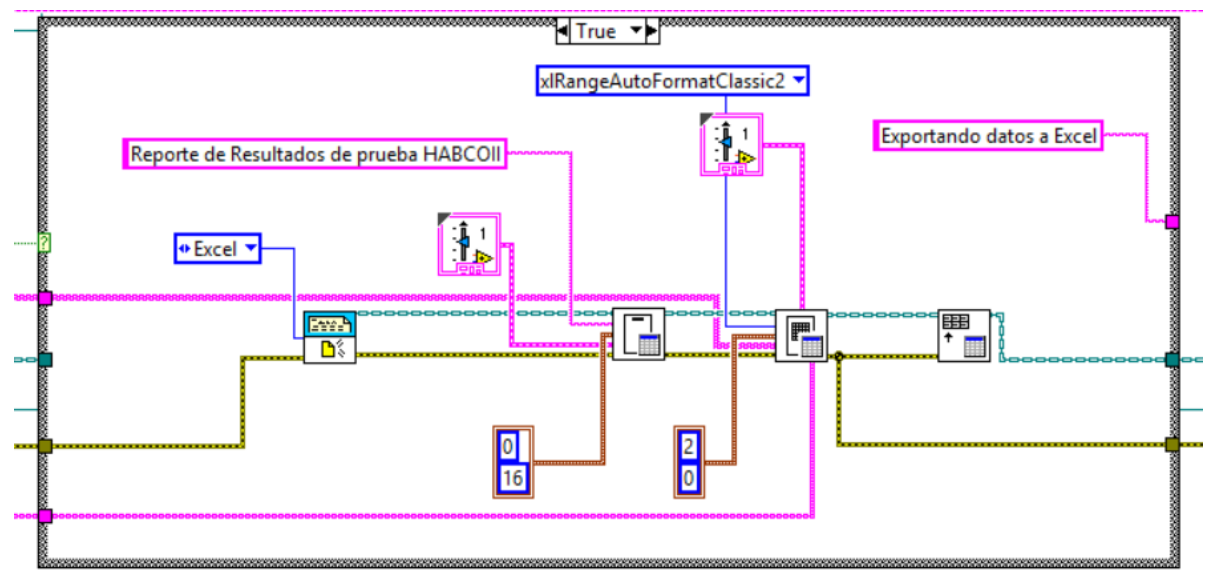

Fig. 14. Creación del reporte de calificación final.

\section{Resultados}

El resultado es un software que de manera amigable permite al usuario la calificación automática de sus pruebas de alveolos en un tiempo de aproximadamente un minuto para una cantidad de setenta y cinco exámenes, ahorrando una gran cantidad de tiempo y desgaste del docente, además de eliminar el error humano.

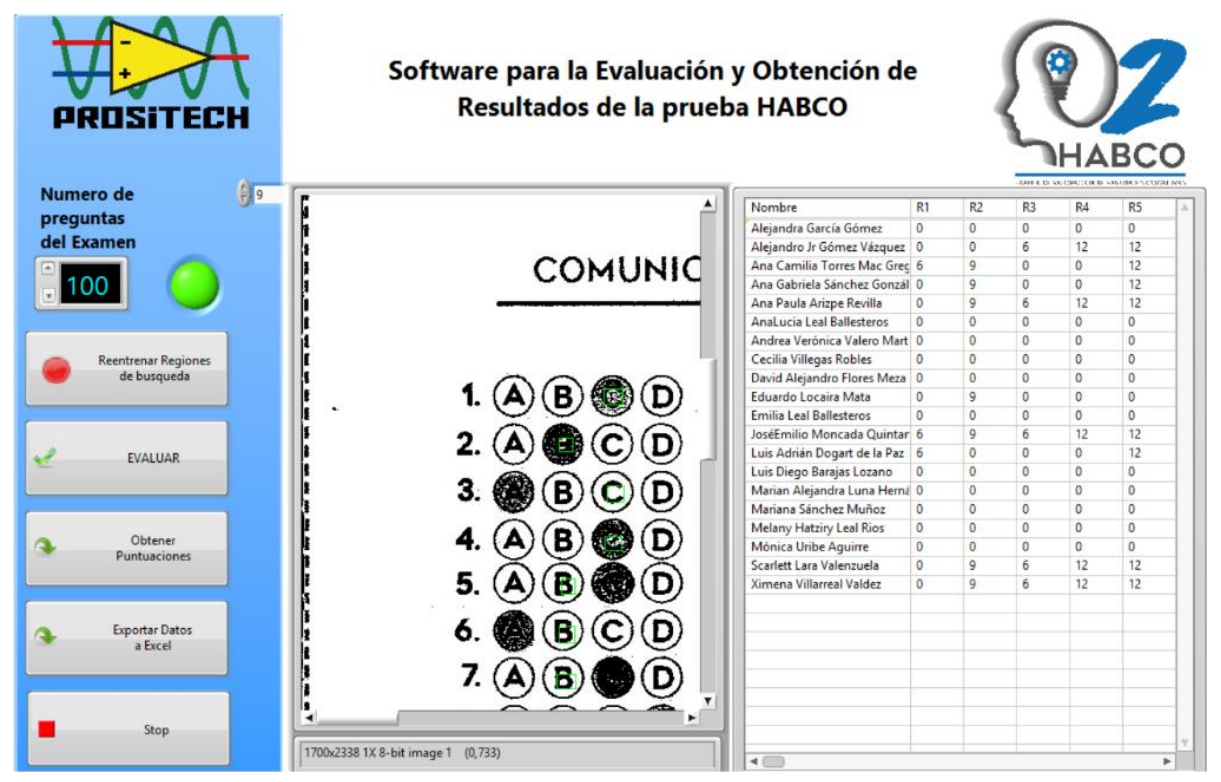

Fig. 15. Interfaz de Usuario.

La flexibilidad del mismo permite su uso para cualquier formato de examen, donde solo requiere la calibración inicial para definir las regiones de interés o de búsqueda de 
respuestas correctas y la definición del patrón de búsqueda de respuesta contestada. No importa el formato de imagen con que se guarda después de la digitalización de cada prueba, ya que el sistema automáticamente cambia el formato de la imagen a 8 bits a blanco y negro para eficientar el uso de los algoritmos de visión utilizados. La interfaz de usuario resultante se muestra en la figura 15.

La etapa de prueba y validación del software se está haciendo en la empresa Reingeniería Didáctica S. de R.L de C.V. y en la empresa Prositech Tecnología Aplicada S.A. de C.V. donde a la fecha se han calificado más de 400 exámenes de valoración de aptitudes de comunicación y habilidad matemática denominado HABCOII diseñado por expertos en pedagogía de Reingeniería Didáctica que sirve como examen de admisión y ubicación en el Colegio Americano de Saltillo (COLAM). La figura 16 muestra un ejemplo de la generación de reporte que arroja el software de los datos generados. Estos resultados son posteriormente interpretados para obtener la información que se requiera.

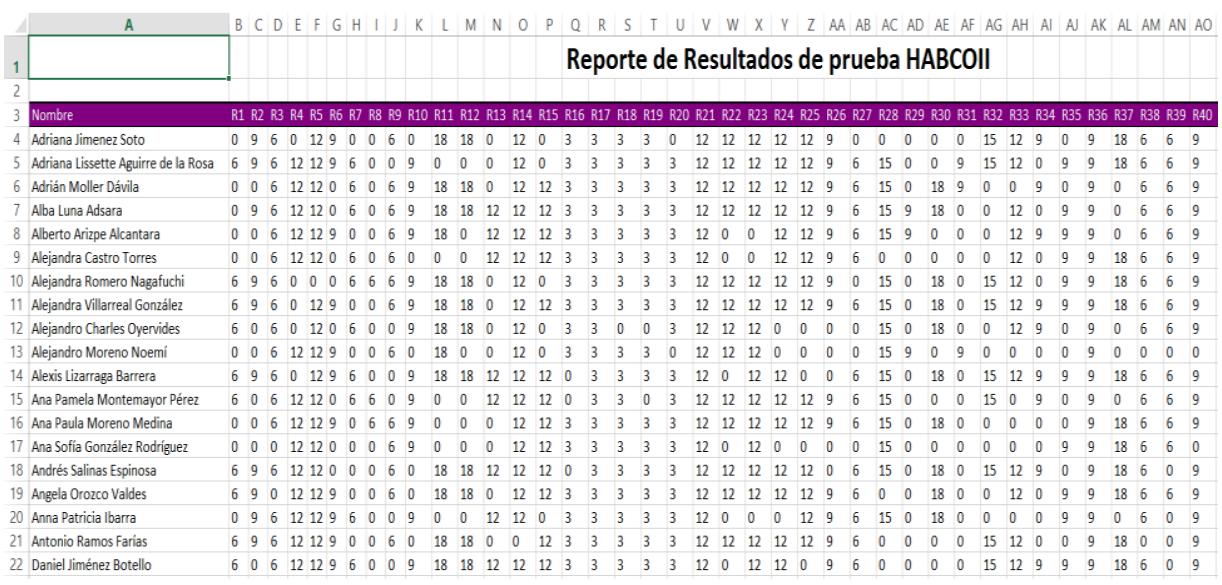

Fig. 16. Reporte en Microsoft Excel generado por el software.

\section{Conclusiones}

La implementación del desarrollo de Software para la solución de problemas es una herramienta poderosa que contribuye a la eficiencia de procesos, ya que al automatizar integrando diferentes tecnologías tal como conectividad ActiveX, algoritmos de análisis de imágenes y señales, contenedores .NET, protocolos de comunicación entre otros, permite mejorar tiempos de producción y control de calidad.

Se desarrolló una herramienta de bajo costo que no requiere hardware especial para su funcionamiento, ya que funciona en cualquier PC bajo ambiente Windows y los exámenes pueden ser digitalizados con cualquier escáner, esto permite al usuario una mejor planificación de su tiempo al contar con una herramienta auxiliar en una labor fundamental como lo es la evaluación y calificación de pruebas académicas, psicométricas o de cualquier tipo que involucre el formato de opción múltiple por medio de alveolos. 
HABCO: Herramienta informática para la automatización de la calificación de exámenes ...

\section{Referencias}

1. National Instruments: NI IMAQ Documentation. June Edition. http://zone.ni.com/reference/en-X/help/370281P01/imaqvision/imaq_light_meter_ rectangle/ (2015)

2. National Instruments: NI IMAQ Documentation. Edition. http://zone.ni.com/reference/enXX/help/370281U-01/imaqvision/imaq_learn_pattern_4/ (2015)

3. National Instruments: LabVIEW 2015 Connectivity User Manual. Edition (2015)

4. Microsoft SQL Server Express MSDN https://msdn.microsoft.com/enus/ sqlserver2014express.aspx (2015)

5. National Instruments: LabVIEW 2015 User Manual. June, Edition (2015) 